\title{
Podolsky propagator in the gap and bound-state equations
}

\author{
Bruno El-Bennich, ${ }^{1, *}$ German Ramos-Zambrano $\odot^{2, \dagger}$ and Eduardo Rojas $\odot^{2, \hbar}$ \\ ${ }^{1}$ Laboratório de Física Teórica e Computacional, Universidad Cidade de São Paulo, \\ Rua Galvão Bueno 868, 01506-000 São Paulo, SP, Brazil \\ ${ }^{2}$ Departamento de Física, Universidad de Nariño, A.A. 1175 San Juan de Pasto, Colombia
}

(Received 4 November 2020; accepted 26 February 2021; published 12 April 2021)

\begin{abstract}
Based on the generalized quantum electrodynamics expression for the Podolsky propagator, which preserves gauge invariance for massive photons, we propose a model for the massive gluon propagator that reproduces well-known features of established strong-interaction models in the framework of the DysonSchwinger equation. By adjusting the Podolsky mass and the coupling strength we thus construct a model with simple analytical properties known from perturbative theory, yet well suited to describe a confining interaction. We obtain solutions of the Dyson-Schwinger equation for the quark at spacelike momenta on the real axis as well as on the complex plane and solving the bound-state problem with the Bethe-Salpeter equation yields masses and weak decay constants of the $\pi, K$ and $\eta_{c}$ in excellent agreement with experimental values, while the $D$ and $D_{s}$ are reasonably well described. The analytical simplicity of this effective interaction has the potential to be useful for phenomenological applications and may facilitate calculations in Minkowski space.
\end{abstract}

DOI: 10.1103/PhysRevD.103.076008

\section{INTRODUCTION}

Mandelstam's seminal work [1] established that the rainbow-ladder truncation of the gap and bound-state equations is an adequate approximation to describe dynamical chiral symmetry breaking (DCSB) [2-12] in quantum chromodynamics (QCD). Later on, within this same truncation scheme, Munczek and Nemirovsky succeeded in reproducing the masses of pseudoscalar and vector meson ground states [13]. More sophisticated models succeeded in the following decades that satisfy theoretical and phenomenological constraints [14-26]. Their popularity owes to a wide range of successful applications to mesons, baryons, hyperons, their excited states and parity partners [22-49].

In this work we propose a model that describes effectively a massive gluon interaction in the infrared region, where we are inspired by the functional structure of generalized quantum electrodynamics (GQED) proposed long ago by Podolsky [50,51]. Historically, this generalization aimed at remedying pathologies inherent to the

\footnotetext{
*bruno.bennich@cruzeirodosul.edu.br †ramoszge@gmail.com *eduro4000@gmail.com
}

Published by the American Physical Society under the terms of the Creative Commons Attribution 4.0 International license. Further distribution of this work must maintain attribution to the author(s) and the published article's title, journal citation, and DOI. Funded by SCOAP.
Maxwell theory and consisted in introducing higher-order derivatives in the Lagrangian of electrodynamics, maintaining at the same time linearity of the equations of motion in the fields. In other words, the goal was to eliminate the infinities that arise in higher-order corrections of point charges and the associated coupling.

However, since this extension of the Lagrangian preserves gauge invariance in a consistent treatment [52,53], GQED has come to be viewed more as a prototype of a theory that contains massless as well as massive photons that do not break gauge invariance. This is because Podolsky's extension of electrodynamics is the only possible linear, Lorentz and $U(1)$ invariant generalization of the Maxwell theory [54] and a consistent quantization of GQED was shown to require a generalized Landau gauge condition [53], while the proper covariant quantization of GQED in this generalized gauge was obtained with functional methods in Ref. [55]. More recently, the Podolsky approach to QED was also reinterpreted as a natural way of providing a Pauli-Villars regularization in ordinary QED [56]. GQED introduces therefore in a consistent manner a mass parameter $m_{P}$ in the vector-boson propagator while preserving gauge invariance and acting as an effective ultraviolet cutoff in Landau gauge.

These features are clearly attractive for modeling the nonperturbative gluon interaction in an Abelianized truncation of QCD, given the compelling body of work that evidence an infrared-finite gluon propagator [57-79] and which can be related to an effective gluon mass. It turns out that in Landau gauge and in the leading truncation of the 
quark's Dyson-Schwinger equation (DSE) we may interpret the Podolsky propagator as a nonperturbative model for the gluon propagator, at least in the low-momentum region, where its massive "dressing function" effectively drives the strength of the DCSB.

In Sec. II we introduce the DSE that describes the quarkgap equation with a Podolsky propagator in Landau gauge and obtain its solutions for different flavors on the spacelike real axis. The functional behavior of the quark's mass and wave-renormalization function is reminiscent of that found with the Maris-Tandy [24] or Qin-Chang [26] models and the obvious question arises whether this interaction is useful for hadron phenomenology. We solve the BetheSalpeter equation (BSE) as usual in Euclidean space to find antiquark-quark bound states, which implies that the arguments of the quark propagators are complex-valued momenta. To obtain the quark propagators on the complex plane, we apply Cauchy's integral theorem that requires
DSE solutions on a contour defined by a parabola describing the complex-momentum distribution $[80,81]$. We note that the convergence of the DSE on such a contour is not generally guaranteed for a given interaction regardless of its convergence on the real axis. Nonetheless, using the Podolsky propagator with an appropriate parametrization the DSE converges rapidly on this contour and the BSE solutions reproduce the mass spectrum and weak decay constants of pseudoscalar mesons as we discuss in Sec. III. We finish with some concluding remarks about possible extensions and applications of the Podolsky propagator in Sec. IV.

\section{DYSON-SCHWINGER EQUATION}

The gap equation for a quark of flavor $f$ is expressed by a DSE for the inverse propagator in Minkowski space as

$$
S_{f}^{-1}(p)=Z_{2} \gamma \cdot p-Z_{4} m_{f}(\mu)-Z_{1} g^{2} \int^{\Lambda} \frac{d^{4} k}{(2 \pi)^{4}} G^{\mu \nu}(q) \frac{\lambda^{a}}{2} \gamma_{\mu} S_{f}(k) \frac{\lambda^{a}}{2} \Gamma_{\nu}(k, p),
$$

where $Z_{2}(\mu, \Lambda), Z_{4}(\mu, \Lambda)$ and $Z_{1}(\mu, \Lambda)$ are the wave function, mass and vertex renormalization constants, respectively. Moreover, $\Gamma_{\mu}^{a}(k, p)=\frac{1}{2} \lambda^{a} \Gamma_{\mu}(k, p)$ is the quarkgluon vertex and $\lambda^{a}$ are the $\mathrm{SU}(3)$ color matrices in the fundamental representation, while $\Lambda$ is a Poincaré-invariant regularization scale, chosen such that $\Lambda \gg \mu$. In GQED the vector-boson propagator in a covariant gauge with gaugefixing parameter $\xi$ and momentum, $q=k-p$, is given by

$$
\begin{aligned}
G_{\mu \nu} & =-i \Delta\left(q^{2}\right) P_{\mu \nu}(q), \\
P_{\mu \nu}(q) & =\Delta_{\mu \nu}(q)-\left[g_{\mu \nu}+(1-\xi) \frac{q_{\mu} q_{\nu}}{q^{2}-m_{P}^{2}}\right] \frac{1}{q^{2}-m_{P}^{2}}+(1-2 \xi) \frac{q_{\mu} q_{\nu}}{q^{2}\left(q^{2}-m_{P}^{2}\right)}+\frac{q_{\mu} q_{\nu}}{\left(q^{2}-m_{P}^{2}\right)^{2}},
\end{aligned}
$$

with the standard gauge-boson propagator,

$$
\Delta_{\mu \nu}(q)=\left[g_{\mu \nu}-(1-\xi) \frac{q_{\mu} q_{\nu}}{q^{2}}\right] \frac{1}{q^{2}}
$$

In general, the Dirac structure of the fermion propagator is fully defined by two covariants and associated scalar functions, the wave-function renormalization $\mathcal{F}_{f}(p)$ and the mass function $\mathcal{M}_{f}(p)$, so that

$$
S_{f}(p)=\frac{\mathcal{F}_{f}(p)}{\gamma \cdot p-\mathcal{M}_{f}(p)} .
$$

In order to determine the renormalization constants and to make quantitative matching with $\mathrm{pQCD}$, one imposes the renormalization conditions,

$$
\left.\mathcal{F}_{f}\left(p^{2}\right)\right|_{p^{2}=\mu^{2}}=1,\left.\quad S_{f}^{-1}(p)\right|_{p^{2}=\mu^{2}}=\gamma \cdot p-m_{f}(\mu),
$$

where $\mu^{2} \gg \Lambda_{\mathrm{QCD}}^{2}$ and $m_{f}(\mu)$ is the renormalized running quark mass; in particular, $m_{f}(\mu)$ is nothing else but the dressed-quark mass function evaluated at one particular deep spacelike point, $p^{2}=\mu^{2}$, namely $m_{f}(\mu)=\mathcal{M}_{f}(\mu)$.

The mass function $\mathcal{M}_{f}\left(p^{2}\right)$ and the renormalization wave function $\mathcal{F}_{f}$ can be projected out from the DSE (1) and rewritten in Euclidean space one obtains ${ }^{1}$ in Landau gauge and for a bare vertex, $\Gamma_{\nu}(k, p)=\gamma_{\nu}$, the two coupled, nonlinear integral equations,

$$
\frac{\mathcal{M}_{f}\left(p_{E}\right)}{\mathcal{F}_{f}\left(p_{E}\right)}=Z_{4} m(\mu)+3 C_{F} \int \frac{d^{4} k_{E}}{(2 \pi)^{4}} \frac{\mathcal{M}_{f}\left(k_{E}\right) \mathcal{F}_{f}\left(k_{E}\right)}{k_{E}^{2}+\mathcal{M}_{f}^{2}\left(k_{E}\right)} \frac{\mathcal{G}\left(q_{E}^{2}\right)}{q_{E}^{2}},
$$

\footnotetext{
${ }^{1}$ See Appendix for details of the calculation in arbitrary covariant gauge.
} 


$$
\frac{1}{\mathcal{F}_{f}\left(p_{E}\right)}=Z_{2}+\frac{C_{F}}{p_{E}^{2}} \int \frac{d^{4} k_{E}}{(2 \pi)^{4}} \frac{\mathcal{F}_{f}\left(k_{E}\right)}{k_{E}^{2}+\mathcal{M}_{f}^{2}\left(k_{E}\right)}\left[3 p_{E} \cdot k_{E}+\frac{2\left[\left(p_{E} \cdot k_{E}\right)^{2}-p_{E}^{2} k_{E}^{2}\right]}{q_{E}^{2}}\right] \frac{\mathcal{G}\left(q_{E}^{2}\right)}{q_{E}^{2}}
$$

We define an interaction model by

$$
\mathcal{G}\left(q_{E}^{2}\right)=4 \pi \alpha_{\mathrm{eff}} \frac{m_{P}^{2}}{q_{E}^{2}+m_{P}^{2}},
$$

with the gluon momentum, $q_{E}=k_{E}-p_{E}$, and the numerical values $\alpha_{\text {eff }}=Z_{1} g^{2} / 4 \pi=7.69$ and $m_{P}^{2}=0.6 \mathrm{GeV}^{2}$. These values have been chosen to reproduce the pion's mass and weak decay constant, as will be discussed in Sec. III. Note that once fixed, these same parameters are employed for other mesons. In our approach we set $\Delta\left(q^{2}\right)=1$ in Eq. (2), which is the perturbative value, such that the gluon-dressing function is defined implicitly by Eq. (8) and thus parametrized by $m_{P}$ and $g^{2}$. As can be appreciated from Fig. 1, the mass functions, $\mathcal{M}_{f}$, and wave-renormalization functions, $\mathcal{F}_{f}$, obtained with this model compare well with the functional form that results from the rainbow-ladder model introduced in Ref. [26], though $\mathcal{F}_{f}$ is more suppressed with the Podolsky model. This is in particular the case for the lighter quark flavors. Likewise, the mass functions are also suppressed in the momentum region $p^{2} \lesssim 1 \mathrm{GeV}^{2}$.

We note that the Podolsky mass, $m_{P} \approx 0.77 \mathrm{GeV}$, which serves as an infrared mass scale in our model, agrees with other gluon mass scales $[14,15,82]$. In particular, in Landau gauge, the integral expressions for $\mathcal{M}_{f}\left(p_{E}\right)$ and $\mathcal{F}_{f}\left(p_{E}\right)$ in Eqs. (6) and (7) can also be obtained by taking the projections and traces of the DSE (1) using a modified perturbative gluon propagator with a mass term:

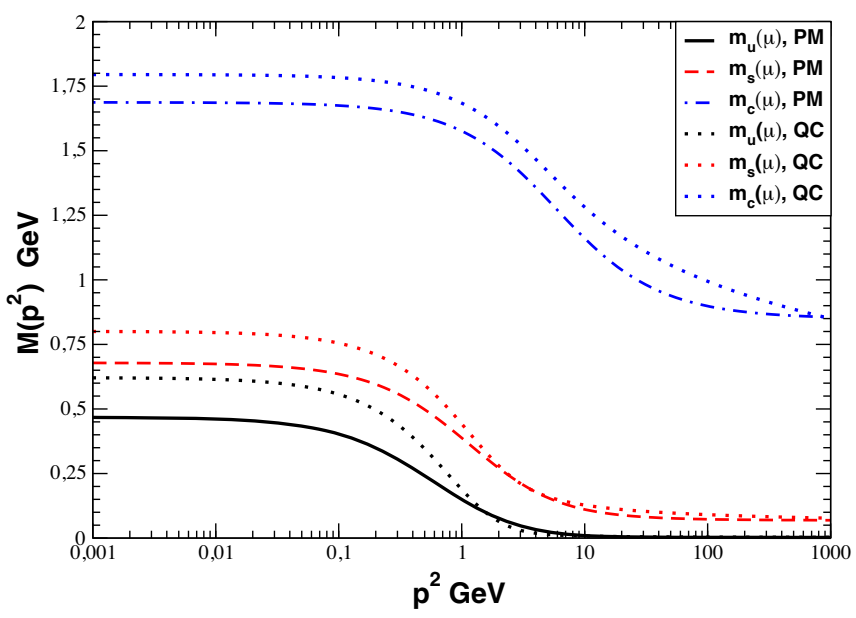

$D_{\mu \nu}(q):=\frac{m_{P}^{2}}{q_{E}^{2}+m_{P}^{2}}\left(g_{\mu \nu}-\frac{q_{E \mu} q_{E \nu}}{q_{E}^{2}}\right) \frac{1}{q_{E}^{2}}=\frac{m_{P}^{2}}{q_{E}^{2}+m_{P}^{2}} \Delta_{\mu \nu}^{\xi=0}\left(q_{E}^{2}\right)$.

Indeed, for $\xi=0$ and with $\Delta\left(q^{2}\right)=1$ the Podolsky propagator (2) reduces to Eq. (9) and is equivalent to multiplying by a factor $m_{P}^{2} / q_{E}^{2}$ the massive gluon propagator introduced in Ref. [14]. The latter is motivated by soft breaking of Becchi-Rouet-Stora-Tyutin (BRST) symmetry and described by a gluon-mass term in the Lagrangian corresponding to a particular case of the Curci-Ferrari model [83]. Alternatively, here the vector-boson mass $m_{P}$ originates in higher-order derivatives which preserve gauge symmetry in a generalized Lagrangian of the Abelian field theory. The coupling $\alpha_{\text {eff }}$, on the other hand, cannot be directly compared with the running strong coupling at this scale. This is because we merely employ the rainbow truncation of the DSE in which $\alpha_{\text {eff }}$ partially accounts for the lack of DCSB from a fully dressed quark-gluon vertex [84-100].

In this context, we remind that one can infer qualitative and analytic properties of the interaction kernel from the mass and wave-renormalization functions via an inversion process of the DSE $[87,88]$ which allows for the comparison of different models. We also stress that it is possible to modify the interaction (8) to include the perturbative $\sim 1 / q^{2}$ running at larger momenta. However, our aim here merely consists in verifying the simple expression's (8) capacity to yield the adequate DCSB observed in hadron phenomenology.

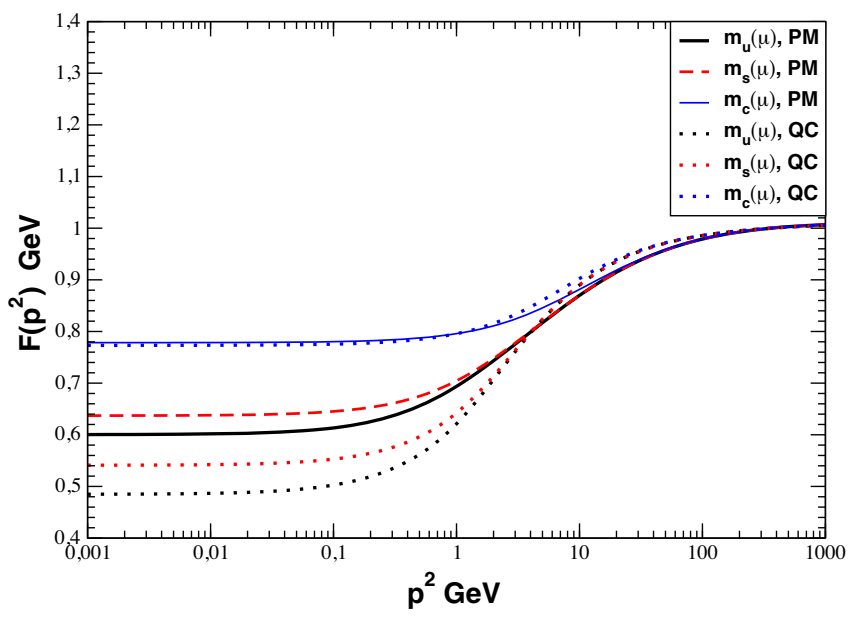

FIG. 1. Mass function, $\mathcal{M}_{f}\left(p^{2}\right)$, and wave function renormalization, $\mathcal{F}_{f}\left(p^{2}\right)$, for the light, strange and charm quarks obtained with Eqs. (6) and (7) using the Podolsky model (PM) (8) compared to DSE solutions calculated with the Qin-Chang (QC) model in Ref. [26]. All functions are renormalized at $\mu=19 \mathrm{GeV}$ with $m_{u, d}(19 \mathrm{GeV})=2.64 \mathrm{MeV}, m_{s}(19 \mathrm{GeV})=70 \mathrm{MeV}$ and $m_{c}(19 \mathrm{GeV})=865 \mathrm{MeV}$. 

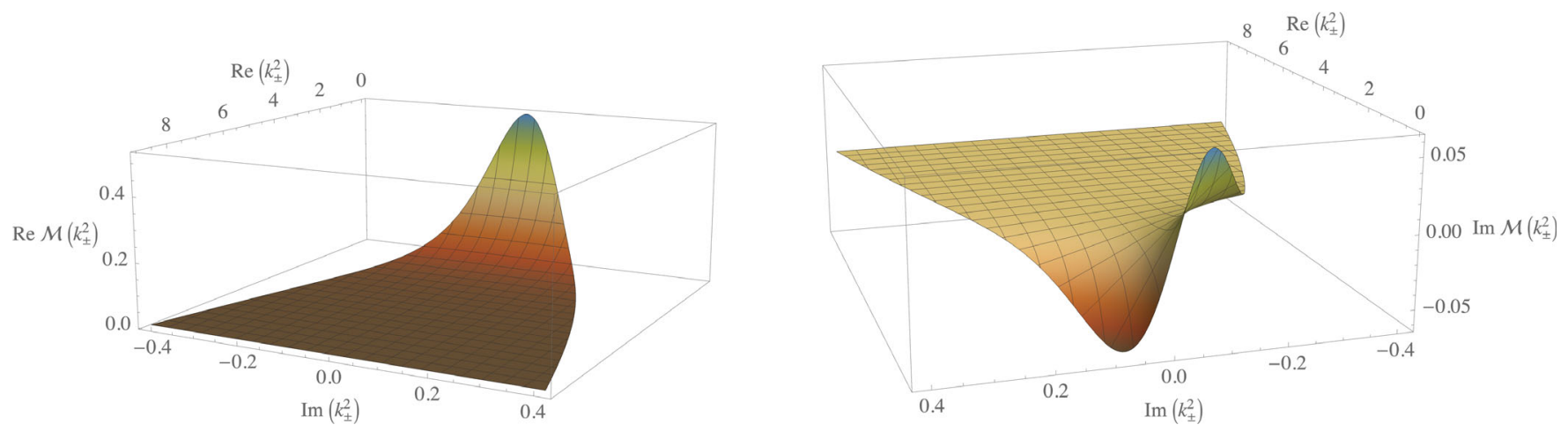

FIG. 2. Real part (left panel) and imaginary part (right panel) of the mass function $\mathcal{M}_{u}\left(p^{2}\right)$ for a current-quark mass $m(19 \mathrm{GeV})=$ $2.64 \mathrm{MeV}$ and an external pion mass $P^{2}=-M_{\pi}^{2}$ using the Podolsky model (8). The parabolic area on the complex $k_{ \pm}^{2}$ plane (in $\mathrm{GeV}^{2}$ ) is defined by the quark momentum in the BSE for a pion as in Eq. (10).

We close this section with the graphs of $\mathcal{M}_{u}\left(k^{2}\right)$ and $\mathcal{F}_{u}\left(k^{2}\right)$ functions on the complex plane, i.e., their solutions calculated on a parabola of complex momenta $p^{2}$ defined by the arguments of the quark propagators in the BSE (11),

$$
k_{ \pm}^{2}=\left(k \pm \eta_{ \pm} P\right)^{2}=k^{2}-\eta_{ \pm}^{2} M_{P}^{2} \pm 2 i \eta_{ \pm} M_{P}|k| z_{k},
$$

where $P^{2}=-M_{P}^{2}$ is the external meson mass of the pseudoscalar meson, $z_{k}=k \cdot P /|k||P|,-1 \leq z_{k} \leq+1$, is an angle and henceforth the Euclidean metric is implicit: $k^{2}=k_{E}^{2}$. The real and imaginary parts of $\mathcal{M}_{u}\left(k^{2}\right)$ and $\mathcal{F}_{u}\left(k^{2}\right)$ are plotted in Figs. 2 and 3, respectively. The behavior of the real part of $\mathcal{M}_{u}\left(k^{2}\right)$ is smooth, monotonically decreasing in both, real and imaginary $k_{ \pm}^{2}$ directions, whereas the real part of $\mathcal{F}_{u}\left(k^{2}\right)$ tends towards its perturbative limit both on and off the real axis. The imaginary part of both functions is characterized by complex-conjugate extrema near the origin of the parabola. For details of the DSE solutions on the complex plane we refer to Refs. [30,80,81]. Within the complex momentum range considered herein, the Podolsky model results in complex DSE solutions that differ somewhat in magnitude but not much in their analytical behavior from the ones obtained

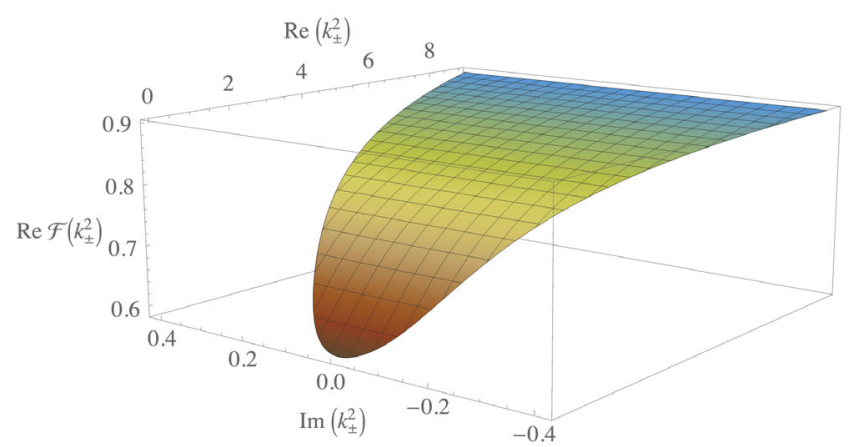

with the gluon model in Ref. [26]. However, to really compare the analytic properties of these models, a more detailed study on the complex plane is necessary that allows to trace poles and branch cuts outside the parabolic contour presented in Figs. 2 and 3.

\section{PSEUDOSCALAR MESON MASSES AND DECAY CONSTANTS}

The homogeneous BSE for a $\bar{q} q$ bound state with relative momentum $p$ and total momentum $P$ can be written as

$\Gamma_{m n}^{f g}(p, P)=\int \frac{d^{4} k_{E}}{(2 \pi)^{4}} \mathcal{K}_{m n}^{k l}(p, k, P)\left[S_{f}\left(k_{+}\right) \Gamma^{f g}(k, P) S_{g}\left(k_{-}\right)\right]_{l k}$

where $m, n, k, l$ collect Dirac and color indices, $f, g$ are flavor indices and $k_{+}=k+\eta_{+} P, k_{-}=k-\eta_{-} P$; $\eta_{+}+\eta_{-}=1$. Since we work within the rainbow-ladder truncation, the BSE kernel is given by

$\mathcal{K}_{m n}^{k l}(p, k, P)=-4 \pi \alpha_{\mathrm{eff}}\left(\frac{\lambda^{a}}{2} \gamma_{\mu}\right)_{k n} P_{\mu \nu}(q)\left(\frac{\lambda^{a}}{2} \gamma_{\nu}\right)_{m l}$

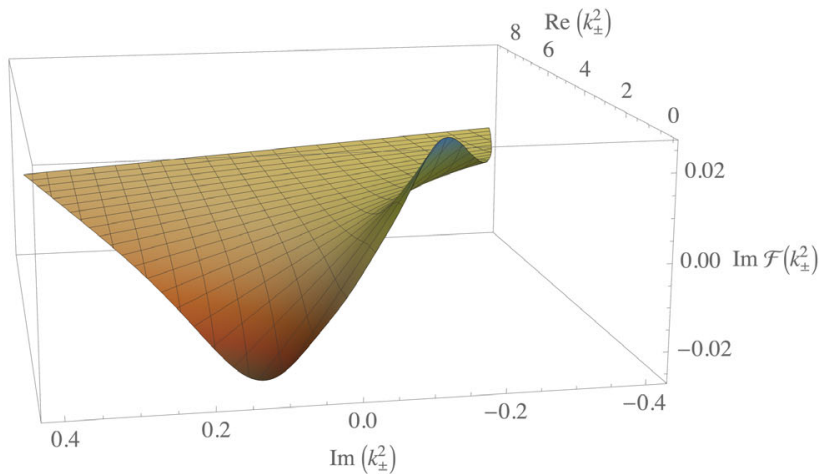

FIG. 3. Real part (left panel) and imaginary part (right panel) of the wave-renormalization function $\mathcal{F}_{u}\left(p^{2}\right)$ for a current-quark mass $m(19 \mathrm{GeV})=2.64 \mathrm{MeV}$ and an external pion mass $P^{2}=-M_{\pi}^{2}$ using the Podolsky model (8). 
which satisfies the axial-vector Ward-Green-Takahashi identity [23] and as a consequence ensures a massless pion in the chiral limit. As such, Eqs. (11) and (12) define an eigenvalue problem with physical solutions at the onshell points, $P^{2}=-M_{P}^{2}$. As in the DSE (1), the vertex renormalization is absorbed in $\alpha_{\text {eff }}$.
The general Poincaré-invariant form of the BetheSalpeter amplitudes (BSA), i.e., the solutions of Eq. (11), for the pseudoscalar channel $J^{P}=0^{-}$in a nonorthogonal base with respect to the Dirac trace, $\mathcal{A}^{\alpha}(p, P)=$ $\gamma_{5}\left\{i \mathbb{I}_{D}, \gamma \cdot P, \gamma \cdot p(p \cdot P), \sigma_{\mu \nu} p_{\mu} P_{\nu}\right\}$, is given by

$$
\Gamma(p, P)=\gamma_{5}\left[i \mathbb{I}_{D} E(p, P)+\gamma \cdot P F(p, P)+\gamma \cdot p p \cdot P G(p, P)+\sigma_{\mu \nu} p_{\mu} P_{\nu} H(p, P)\right]
$$

where we suppress color, Dirac and flavor indices for the sake of readability and $\sigma_{\mu \nu}=i / 2\left[\gamma_{\mu}, \gamma_{\nu}\right]$. The functions $\mathcal{F}_{f}^{\alpha}(p, P)=\{E(p, P), F(p, P), G(p, P), H(p, P)\}$ are Lorentz-invariant scalar amplitudes. For sake of completeness, we note that all BSA are normalized canonically as

$$
2 P_{\mu}=\int^{\Lambda} \frac{d^{4} k_{E}}{(2 \pi)^{4}} \operatorname{Tr}_{\mathrm{CD}}\left[\bar{\Gamma}(k,-P) \frac{\partial S\left(k_{+}\right)}{\partial P_{\mu}} \Gamma(k, P) S\left(k_{-}\right)+\bar{\Gamma}(k,-P) S\left(k_{+}\right) \Gamma(k, P) \frac{\partial S\left(k_{-}\right)}{\partial P_{\mu}}\right],
$$

where we omit a third term that stems from the derivative of the kernel, $\partial \mathcal{K}_{m n}^{k l}(p, k, P) / \partial P_{\mu}$, since it does not contribute in the rainbow-ladder truncation of Eq. (12). ${ }^{2}$ In Eq. (14), the charge-conjugated BSA is defined as $\bar{\Gamma}(k,-P):=$ $C \Gamma^{T}(-k,-P) C^{T}$, where $C$ is the charge conjugation operator and the trace is over Dirac and color indices. With this normalization we obtain the meson's leptonic decay constants via the integral,

$f_{P} P_{\mu}=\int^{\Lambda} \frac{d^{4} k_{E}}{(2 \pi)^{4}} \operatorname{Tr}_{\mathrm{CD}}\left[\gamma_{5} \gamma_{\mu} S\left(k_{+}\right) \Gamma(k, P) S\left(k_{-}\right)\right]$.

The BSE (11) is calculated in Euclidean space and therefore the momenta $k_{+}$and $k_{-}$of the quark propagators are complex valued. We follow the numerical prescriptions introduced in Ref. [80] and refined in Refs. [30,81] in computing solutions of the DSE in a parabola on the complex plane.

The potential of this effective interaction is illustrated for the pseudoscalar $\bar{q} q$ channel in Table I. We work in the isospin-symmetric limit $m_{u}=m_{d}$ and set the light quark's mass scale at $19 \mathrm{GeV}$ with the pion mass; analogously we fix the strange- and charm-quark masses with the kaon and the $\eta_{c}$. The resulting weak decay constant of the pion is within $2 \%$ of the experimentally extracted value and that of the kaon is within $5 \%$ of its reference value. The charmonium's decay constant is about $15 \%$ larger than a calculation using lattice QCD. We also compare the weak decay constants obtained with Eq. (15) with those making use of the Gell-Mann-Oaks-Renner (GMOR) relation described in

\footnotetext{
${ }^{2}$ We verify the values obtained with Eq. (14) with the equivalent normalization condition [101,102]: $\left(d \ln \lambda_{n} / d P^{2}\right)^{-1}=$ $\operatorname{tr} \int_{k}^{\Lambda} 3 \bar{\Gamma}(k,-P) S\left(k_{+}\right) \Gamma(k, P) S\left(k_{-}\right)$.
}

detail, for example, in Ref. [30] and find very good agreement.

This is a first step to establish the model's potential to correctly describe light-meson and quarkonia ground states and as an additional check we calculate the $D$ and $D_{s}$ meson's masses and decay constants. As observed in Table I and previously in Ref. [30], their masses are overestimated by $12 \%$ for the $D$ and $8 \%$ for the $D_{s}$ with

TABLE I. Mass spectrum and decay constants for flavor singlet and nonsinglet $J^{P}=0^{-}$mesons in GeV. We adjusted the current masses to $m_{u}=m_{d}=2.64 \mathrm{MeV}, m_{s}=70 \mathrm{MeV}$ and $m_{c}=$ $865 \mathrm{MeV}$ in order to reproduce the ground-state masses of the $\pi, K$ and $\eta$ mesons, respectively. The weak decay constant is obtained with Eq. (15) and the appropriate GMOR relation. For comparison, we reproduce the values in Ref. [30] using the QinChang (QC) interaction model of Ref. [26].

\begin{tabular}{lccc}
\hline \hline & Podolsky model & Q.-C. model & Reference \\
\hline$M_{\pi}$ & 0.138 & 0.138 & $0.139[103]$ \\
$f_{\pi}$ & 0.133 & 0.139 & $0.130[103]$ \\
$f_{\pi}^{\text {GMOR }}$ & 0.117 & & \\
$M_{K}$ & 0.494 & 0.493 & $0.493[103]$ \\
$f_{K}$ & 0.164 & 0.164 & $0.156[103]$ \\
$f_{K}^{\mathrm{GMOR}}$ & 0.162 & 0.162 & \\
$M_{\eta_{c}(1 S)}$ & 2.985 & 3.065 & $2.984[103]$ \\
$f_{\eta_{c}(1 S)}$ & 0.454 & 0.389 & $0.395[104]$ \\
$f_{\eta_{c}(1 S)}^{\mathrm{GMOR}}$ & 0.451 & 0.380 & \\
$M_{D}$ & 2.100 & 2.115 & $1.870[103]$ \\
$f_{D}$ & 0.263 & 0.204 & $0.212[105]$ \\
$M_{D_{s}}$ & 2.130 & 2.130 & $1.968[103]$ \\
$f_{D_{s}}$ & 0.304 & 0.249 & $0.250[105]$ \\
\hline \hline
\end{tabular}


respect to experimental values. This is a consequence of the rainbow-ladder truncation which neglects the dramatically different impact of vertex dressing for heavy and light quarks and can be strongly improved by inclusion of this effect [36]. Likewise, the weak decay constants are also $22 \%$ larger than results reported by the FLAG collaboration [105]. Nonetheless, the Podolsky propagator facilitates the numerical treatment of the DSE with a large external Euclidean mass (using $\eta_{+}=0.8$ and $\eta_{-}=0.2$ ) on the complex plane in comparison with the model of Ref. [26] and both iterative treatments of the DSE and BSE mesons converge rapidly in case of the $D$ and $D_{s}$.

\section{SUMMARY AND CONCLUSIONS}

We solve for the first time the DSE with the Podolsky propagator in rainbow truncation and find a mass gap for a typical hadronic scale of the Podolsky mass. Based on this observation, we propose a novel interaction model within the rainbow-ladder truncation of the DSE and BSE kernels, based on the Podolsky propagator which preserves gauge invariance in perturbative GQED. However, in our approach we merely interpret this massive propagator as a nonperturbative ansatz for the dressed gluon propagator. The associated mass scale we find is reminiscent of earlier DSE studies of the gluon. The Podolsky propagator has the same integrability properties as the perturbative propagator, a feature which is the practical motivation for our model, and when employed in the appropriate DSE we find wellbehaved solutions on the complex plane even for larger timelike momenta. Employing these complex solutions for the quark propagators in the BSE, we fix the quark masses and interaction parameters with the masses of the $\pi, K, \eta_{c}$ and find weak decay constants that agree very well with experimental reference values or results from lattice-QCD simulations. The $D$ mesons are also obtained within this framework, where their masses are somewhat overestimated and the mass difference between the $D$ and $D_{s}$ is too small, a consequence of the too simplistic truncation for heavy-light systems.

The Podolsky propagator along with the ansatz in Eq. (8) ought to be used in future calculations of radial excitations and other $J^{\mathrm{PC}}$ channels. Since we are interested in static properties of the mesons, we here omit the perturbative term of the interaction commonly included in other models. As a consequence of this simple form, we established that the angular integration of the DSE, Eqs. (A11) and (A12), can be performed analytically. Likewise, the simple pole structure of the Podolsky propagator allows, in given cases, for analytical calculations as with any perturbative propagator. These features are attractive enough to raise one's curiosity about possible solutions of the DSE and BSE in Minkowski space, which is of practical importance with regard to the study of parton distribution functions defined on the light front. Amongst other applications, one may explore this interaction to obtain more sophisticated quark propagators and wave functions at finite density to understand the pion's properties in a dense nuclear medium [106].

\section{ACKNOWLEDGMENTS}

We acknowledge financial support from "Patrimonio Autónomo Fondo Nacional de Financiamiento para la Ciencia, la Tecnología y la Innovación, Francisco José de Caldas," from Fundação de Amparo à Pesquisa do Estado de São Paulo, Grant No. 2018/20218-4, and Conselho Nacional de Desenvolvimento Científico e Tecnológico, Grant No. 428003/2018-4. This work was also partly supported by the "Vicerrectoría de Investigaciones e Interacción Social VIIS de la Universidad de Nariño," Projects No. 1928 and No. 2172. B.E. appreciated the hospitality of Universidade de Nariño during his stay in San Juan de Pasto and participates in the INCT-FNA Project No. 464898/2014-5. Insightful comments on the manuscript by Fernando Serna were strongly appreciated.

\section{APPENDIX: DYSON-SCHWINGER EQUATION WITH THE PODOLSKY PROPAGATOR}

For a a quark propagator, the DSE in Minkowski space is described by a nonlinear integral equation,

$$
S^{-1}(p)=Z_{2} \gamma \cdot p-Z_{4} m(\mu)+i Z_{1} g^{2} C_{F} \int \frac{d^{4} k}{(2 \pi)^{4}} \gamma_{\mu} S(k) \Gamma_{\nu}(k, p) G^{\mu \nu}(q),
$$

with $C_{F}=4 / 3$ in the fundamental representation of SU(3). The GQED gauge propagator in a covariant gauge, specified by the gauge parameter $\xi$, is given by

$$
G_{\mu \nu}=\Delta\left(q^{2}\right) P_{\mu \nu}(q),
$$

with $q=k-p$ and where

$$
P_{\mu \nu}(q)=\Delta_{\mu \nu}(q)-\left[g_{\mu \nu}+(1-\xi) \frac{q_{\mu} q_{\nu}}{q^{2}-m_{P}^{2}}\right] \frac{1}{q^{2}-m_{P}^{2}}+(1-2 \xi) \frac{q_{\mu} q_{\nu}}{q^{2}\left(q^{2}-m_{P}^{2}\right)}+\frac{q_{\mu} q_{\nu}}{\left(q^{2}-m_{P}^{2}\right)^{2}} .
$$


Here, $\Delta_{\mu \nu}(q)$ is the contribution of the Maxwell theory:

$$
\Delta_{\mu \nu}(q)=\frac{1}{q^{2}}\left(g_{\mu \nu}-\frac{q_{\mu} q_{\nu}}{q^{2}}\right)+\xi \frac{q_{\mu} q_{\nu}}{q^{4}} .
$$

We employ for the vertex structure its bare form, which is the rainbow truncation:

$$
\Gamma_{\mu} \rightarrow \gamma_{\mu}
$$

In general, the Dirac structure of the fermion propagator depends on two independent functions, the wave function renormalization $\mathcal{F}(p)$ and the mass function $\mathcal{M}_{f}(p)$, such that

$$
S(p)=\frac{\mathcal{F}(p)}{\gamma \cdot p-\mathcal{M}(p)} .
$$

With this, expression (A1) can be rewritten as

$$
\frac{\gamma \cdot p-\mathcal{M}(p)}{\mathcal{F}(p)}=Z_{2} \gamma \cdot p-Z_{4} m(\mu)+i Z_{1} g^{2} C_{F} \int \frac{d^{4} k}{(2 \pi)^{4}} \mathcal{F}(k) \gamma_{\mu} \frac{\gamma \cdot k+\mathcal{M}(k)}{k^{2}-\mathcal{M}^{2}(k)} G^{\mu \nu}(q) \gamma_{\nu} .
$$

Taking the trace of Eq. (A7) results in the expression,

$$
\frac{\mathcal{M}(p)}{\mathcal{F}(p)}=Z_{4} m(\mu)-i Z_{1} g^{2} C_{F} \int \frac{d^{4} k}{(2 \pi)^{4}} \frac{\mathcal{M}(k) \mathcal{F}(k)}{k^{2}-\mathcal{M}^{2}(k)} G^{\mu}{ }_{\mu}(q),
$$

where $G^{\mu}{ }_{\mu}(q)=\Delta\left(q^{2}\right) P_{\mu}^{\mu}(q)$ and

$$
\begin{aligned}
P_{\mu}^{\mu}(q) & =\Delta_{\mu}^{\mu}(q)-\left[g_{\mu}^{\mu}+(1-\xi) \frac{q^{\mu} q_{\mu}}{q^{2}-m_{P}^{2}}\right] \frac{1}{q^{2}-m_{P}^{2}}+(1-2 \xi) \frac{q^{\mu} q_{\mu}}{q^{2}\left(q^{2}-m_{P}^{2}\right)}+\frac{q^{\mu} q_{\mu}}{\left(q^{2}-m_{P}^{2}\right)^{2}} \\
& =\Delta_{\mu}^{\mu}(q)-(3+2 \xi) \frac{1}{q^{2}-m_{P}^{2}}+\xi \frac{q^{2}}{\left(q^{2}-m_{P}^{2}\right)^{2}},
\end{aligned}
$$

with

$$
\Delta_{\mu}^{\mu}(q)=\frac{1}{q^{2}}\left(g^{\mu}{ }_{\mu}-\frac{q^{\mu} q_{\mu}}{q^{2}}\right)+\xi \frac{q^{\mu} q_{\mu}}{q^{4}}=(3+\xi) \frac{1}{q^{2}}
$$

It follows that,

$$
\begin{aligned}
\frac{\mathcal{M}(p)}{\mathcal{F}(p)} & =Z_{4} m(\mu)-i Z_{1} g^{2} C_{F} \int \frac{d^{4} k}{(2 \pi)^{4}} \frac{\mathcal{M}(k) \mathcal{F}(k)}{k^{2}-\mathcal{M}^{2}(k)} \Delta\left(q^{2}\right)\left[\Delta^{\mu}{ }_{\mu}(q)-(3+2 \xi) \frac{1}{q^{2}-m_{P}^{2}}+\xi \frac{q^{2}}{\left(q^{2}-m_{P}^{2}\right)^{2}}\right] \\
& =\left.\frac{\mathcal{M}(p)}{\mathcal{F}(p)}\right|_{M}+\left.\frac{\mathcal{M}(p)}{\mathcal{F}(p)}\right|_{P},
\end{aligned}
$$

where the two terms are

$$
\begin{aligned}
\left.\frac{\mathcal{M}(p)}{\mathcal{F}(p)}\right|_{M} & \equiv Z_{4} m(\mu)-i Z_{1} g^{2} C_{F} \int \frac{d^{4} k}{(2 \pi)^{4}} \frac{\mathcal{M}(k) \mathcal{F}(k)}{k^{2}-\mathcal{M}^{2}(k)} \Delta\left(q^{2}\right) \Delta^{\mu}{ }_{\mu}(q) \\
& =Z_{4} m(\mu)-i Z_{1} g^{2} C_{F}(3+\xi) \int \frac{d^{4} k}{(2 \pi)^{4}} \frac{\mathcal{M}(k) \mathcal{F}(k)}{k^{2}-\mathcal{M}^{2}(k)} \frac{\Delta\left(q^{2}\right)}{q^{2}}
\end{aligned}
$$

which is the Maxwell contribution and 


$$
\left.\frac{\mathcal{M}(p)}{\mathcal{F}(p)}\right|_{P} \equiv(3+2 \xi) i Z_{1} g^{2} C_{F} \int \frac{d^{4} k}{(2 \pi)^{4}} \frac{\mathcal{M}(k) \mathcal{F}(k)}{k^{2}-\mathcal{M}^{2}(k)} \frac{\Delta\left(q^{2}\right)}{q^{2}-m_{P}^{2}}-i Z_{1} g^{2} C_{F} \xi \int \frac{d^{4} k}{(2 \pi)^{4}} \frac{\mathcal{M}(k) \mathcal{F}(k)}{k^{2}-\mathcal{M}^{2}(k)} \frac{q^{2} \Delta\left(q^{2}\right)}{\left(q^{2}-m_{P}^{2}\right)^{2}}
$$

is due to Podolsky's GQED extension.

Now, if we multiply Eq. (A7) by $\gamma^{\beta} p_{\beta}$ and take the trace, we can project out the wave function renormalization $\mathcal{F}(p)$, such that

$$
\frac{p^{2}}{\mathcal{F}(p)}=Z_{2} p^{2}+i Z_{1} g^{2} C_{F} \int \frac{d^{4} k}{(2 \pi)^{4}} \mathcal{F}(k) \frac{2 p_{\mu} k_{\nu} P^{\mu \nu}(q)-p \cdot k P^{\mu}{ }_{\mu}(q)}{k^{2}-\mathcal{M}^{2}(k)} \Delta\left(q^{2}\right)
$$

where the expression in the numerator is found to be

$$
\begin{aligned}
2 p_{\mu} k_{\nu} P^{\mu \nu}(q)-p \cdot k P_{\mu}^{\mu}(q)= & {\left[(\xi-3) \frac{(p \cdot k)}{q^{2}}+2(\xi-1) \frac{1}{q^{4}}\left[(p \cdot k)^{2}-p^{2} k^{2}\right]\right] } \\
& +\left[(3-2 \xi)(p \cdot k)+2(1-2 \xi) \frac{(p \cdot k)^{2}-p^{2} k^{2}}{q^{2}}\right] \frac{1}{q^{2}-m_{P}^{2}} \\
& +\left[\xi(p \cdot k) q^{2}+2 \xi\left((p \cdot k)^{2}-p^{2} k^{2}\right)\right] \frac{1}{\left(q^{2}-m_{P}^{2}\right)^{2}}
\end{aligned}
$$

We can again separate the integral into a Maxwell and Podolsky contribution,

$$
\frac{1}{\mathcal{F}(p)}=\left.\frac{1}{\mathcal{F}(p)}\right|_{M}+\left.\frac{1}{\mathcal{F}(p)}\right|_{P}
$$

where

$$
\left.\frac{1}{\mathcal{F}(p)}\right|_{M} \equiv Z_{2}+\frac{i Z_{1} g^{2} C_{F}}{p^{2}} \int \frac{d^{4} k}{(2 \pi)^{4}} \frac{\mathcal{F}(k) \Delta\left(q^{2}\right)}{k^{2}-\mathcal{M}^{2}(k)}\left[(\xi-3) \frac{p \cdot k}{q^{2}}+2(\xi-1) \frac{1}{q^{4}}\left[(p \cdot k)^{2}-p^{2} k^{2}\right]\right],
$$

and

$$
\begin{aligned}
\left.\frac{1}{\mathcal{F}(p)}\right|_{P} \equiv & \frac{i Z_{1} g^{2} C_{F}}{p^{2}} \int \frac{d^{4} k}{(2 \pi)^{4}} \frac{\mathcal{F}(k) \Delta\left(q^{2}\right)}{k^{2}-\mathcal{M}^{2}(k)}\left[(3-2 \xi) p \cdot k+2(1-2 \xi) \frac{(p \cdot k)^{2}-p^{2} k^{2}}{q^{2}}\right] \frac{1}{q^{2}-m_{P}^{2}} \\
& +\frac{i Z_{1} g^{2} C_{F}}{p^{2}} \xi \int \frac{d^{4} k}{(2 \pi)^{4}} \frac{\mathcal{F}(k) \Delta\left(q^{2}\right)}{k^{2}-\mathcal{M}^{2}(k)}\left[p \cdot k q^{2}+2\left\{(p \cdot k)^{2}-p^{2} k^{2}\right\}\right] \frac{1}{\left(q^{2}-m_{P}^{2}\right)^{2}} .
\end{aligned}
$$

\section{Landau gauge}

In Landau gauge, Eq. (A10) takes the form

$$
\begin{gathered}
\left.\frac{\mathcal{M}(p)}{\mathcal{F}(p)}\right|_{M}=Z_{4} m(\mu)-3 i Z_{1} g^{2} C_{F} \int \frac{d^{4} k}{(2 \pi)^{4}} \frac{\mathcal{M}(k) \mathcal{F}(k)}{k^{2}-\mathcal{M}^{2}(k)} \frac{\Delta\left(q^{2}\right)}{q^{2}}, \\
\left.\frac{\mathcal{M}(p)}{\mathcal{F}(p)}\right|_{P}=3 i Z_{1} g^{2} C_{F} \int \frac{d^{4} k}{(2 \pi)^{4}} \frac{\mathcal{M}(k) \mathcal{F}(k)}{k^{2}-\mathcal{M}^{2}(k)} \frac{\Delta\left(q^{2}\right)}{q^{2}-m_{P}^{2}} .
\end{gathered}
$$

If we apply a Wick rotation we obtain the Euclidean space expressions for these integrals,

$$
\left.\frac{\mathcal{M}\left(p_{E}\right)}{\mathcal{F}\left(p_{E}\right)}\right|_{M}=Z_{4} m(\mu)+3 Z_{1} g^{2} C_{F} \int \frac{d^{4} k_{E}}{(2 \pi)^{4}} \frac{\mathcal{M}\left(k_{E}\right) \mathcal{F}\left(k_{E}\right)}{k_{E}^{2}+\mathcal{M}^{2}\left(k_{E}\right)} \frac{\Delta\left(q_{E}^{2}\right)}{q_{E}^{2}}
$$




$$
\left.\frac{\mathcal{M}\left(p_{E}\right)}{\mathcal{F}\left(p_{E}\right)}\right|_{P}=-3 Z_{1} g^{2} C_{F} \int \frac{d^{4} k_{E}}{(2 \pi)^{4}} \frac{\mathcal{M}\left(k_{E}\right) \mathcal{F}\left(k_{E}\right)}{k_{E}^{2}+\mathcal{M}^{2}\left(k_{E}\right)} \frac{\Delta\left(q_{E}^{2}\right)}{q_{E}^{2}+m_{P}^{2}},
$$

and we obtain explicitly

$$
\frac{\mathcal{M}_{f}\left(p_{E}\right)}{\mathcal{F}\left(p_{E}\right)}=Z_{4} m(\mu)+3 Z_{1} g^{2} C_{F} \int \frac{d^{4} k_{E}}{(2 \pi)^{4}} \frac{\mathcal{M}\left(k_{E}\right) \mathcal{F}\left(k_{E}\right)}{k_{E}^{2}+\mathcal{M}^{2}\left(k_{E}\right)}\left(\frac{1}{q_{E}^{2}}-\frac{1}{q_{E}^{2}+m_{P}^{2}}\right) \Delta\left(q_{E}^{2}\right) .
$$

Similarly, Eq. (A14) becomes in Landau gauge

$$
\begin{aligned}
\left.\frac{1}{\mathcal{F}(p)}\right|_{M} & =Z_{2}-\frac{i Z_{1} g^{2} C_{F}}{p^{2}} \int \frac{d^{4} k}{(2 \pi)^{4}} \frac{\mathcal{F}(k)}{k^{2}-\mathcal{M}^{2}(k)}\left[3 p \cdot k+2 \frac{(p \cdot k)^{2}-p^{2} k^{2}}{q^{2}}\right] \frac{\Delta\left(q^{2}\right)}{q^{2}} \\
\left.\frac{1}{\mathcal{F}(p)}\right|_{P} & =\frac{i Z_{1} g^{2} C_{F}}{p^{2}} \int \frac{d^{4} k}{(2 \pi)^{4}} \frac{\mathcal{F}(k)}{k^{2}-\mathcal{M}^{2}(k)}\left[3 p \cdot k+2 \frac{(p \cdot k)^{2}-p^{2} k^{2}}{q^{2}}\right] \frac{\Delta\left(q^{2}\right)}{q^{2}-m_{P}^{2}}
\end{aligned}
$$

In Euclidean space these integrals are given by

$$
\begin{aligned}
& \left.\frac{1}{\mathcal{F}\left(p_{E}\right)}\right|_{M}=Z_{2}+\frac{Z_{1} g^{2} C_{F}}{p_{E}^{2}} \int \frac{d^{4} k_{E}}{(2 \pi)^{4}} \frac{\mathcal{F}\left(k_{E}\right)}{k_{E}^{2}+\mathcal{M}^{2}\left(k_{E}\right)}\left[3 p_{E} \cdot k_{E}+2 \frac{\left(p_{E} \cdot k_{E}\right)^{2}-p_{E}^{2} k_{E}^{2}}{q_{E}^{2}}\right] \frac{\Delta\left(q_{E}^{2}\right)}{q_{E}^{2}}, \\
& \left.\frac{1}{\mathcal{F}\left(p_{E}\right)}\right|_{P}=-\frac{Z_{1} g^{2} C_{F}}{p_{E}^{2}} \int \frac{d^{4} k_{E}}{(2 \pi)^{4}} \frac{\mathcal{F}\left(k_{E}\right)}{k_{E}^{2}+\mathcal{M}^{2}\left(k_{E}\right)}\left[3 p_{E} \cdot k_{E}+2 \frac{\left(p_{E} \cdot k_{E}\right)^{2}-p_{E}^{2} k_{E}^{2}}{q_{E}^{2}}\right] \frac{\Delta\left(q_{E}^{2}\right)}{q_{E}^{2}+m_{P}^{2}} .
\end{aligned}
$$

Adding the two contributions we finally arrive at the Euclidean-space integral equation,

$$
\frac{1}{\mathcal{F}\left(p_{E}\right)}=Z_{2}+\frac{Z_{1} g^{2} C_{F}}{p_{E}^{2}} \int \frac{d^{4} k_{E}}{(2 \pi)^{4}} \frac{\mathcal{F}\left(k_{E}\right)}{k_{E}^{2}+\mathcal{M}^{2}\left(k_{E}\right)}\left[3 p_{E} \cdot k_{E}+2 \frac{\left(p_{E} \cdot k_{E}\right)^{2}-p_{E}^{2} k_{E}^{2}}{q_{E}^{2}}\right]\left[\frac{1}{q_{E}^{2}}-\frac{1}{q_{E}^{2}+m_{P}^{2}}\right] \Delta\left(q_{E}^{2}\right) .
$$

This simplifies to

$$
\frac{1}{\mathcal{F}\left(p_{E}\right)}=Z_{2}+\frac{Z_{1} g^{2} C_{F}}{p_{E}^{2}} \int \frac{d^{4} k_{E}}{(2 \pi)^{4}} \frac{\mathcal{F}\left(k_{E}\right)}{k_{E}^{2}+\mathcal{M}^{2}\left(k_{E}\right)}\left[3 p_{E} \cdot k_{E}+2 \frac{\left(p_{E} \cdot k_{E}\right)^{2}-p_{E}^{2} k_{E}^{2}}{q_{E}^{2}}\right] \frac{m_{P}^{2}}{q_{E}^{2}+m_{P}^{2}} \frac{\Delta\left(q_{E}^{2}\right)}{q_{E}^{2}},
$$

which justifies the definition of $\mathcal{G}\left(q_{E}^{2}\right)$ in Eq. (8) in the particular case of Landau gauge and $\Delta\left(q_{E}^{2}\right)=1$. It turns out that the angular integration of Eqs. (A21) and (A26) can be performed analytically using

$$
\begin{aligned}
p_{E} \cdot k_{E} & =p_{E} k_{E} \cos \theta_{1}, \\
\left(p_{E} \cdot k_{E}\right)^{2}-p_{E}^{2} k_{E}^{2} & =-p_{E}^{2} k_{E}^{2} \sin ^{2} \theta_{1},
\end{aligned}
$$

and working in the rest frame where $p_{E}^{\mu}=\left(p_{E}, 0,0,0\right)$.

[1] S. Mandelstam, Phys. Rev. D 20, 3223 (1979).

[2] J. M. Cornwall, R. Jackiw, and E. Tomboulis, Phys. Rev. D 10, 2428 (1974).

[3] J. M. Cornwall, Phys. Rev. D 26, 1453 (1982).

[4] J. M. Cornwall and J. Papavassiliou, Phys. Rev. D 40, 3474 (1989).

[5] C. J. Burden and C. D. Roberts, Phys. Rev. D 47, 5581 (1993).
[6] N. Brown and M. R. Pennington, Phys. Rev. D 39, 2723 (1989).

[7] C. S. Fischer, R. Alkofer, T. Dahm, and P. Maris, Phys. Rev. D 70, 073007 (2004).

[8] A. Bashir and A. Raya, Few-Body Syst. 41, 185 (2007).

[9] L. Chang, I. C. Cloët, B. El-Bennich, T. Klähn, and C. D. Roberts, Chin. Phys. C 33, 1189 (2009). 
[10] A. Bashir, A. Raya, and J. Rodríguez-Quintero, Phys. Rev. D 88, 054003 (2013).

[11] A. Bashir, L. Chang, I. C. Cloët, B. El-Bennich, Y. X. Liu, C. D. Roberts, and P. C. Tandy, Commun. Theor. Phys. 58, 79 (2012).

[12] I. C. Cloët and C. D. Roberts, Prog. Part. Nucl. Phys. 77, 1 (2014).

[13] H. J. Munczek and A. M. Nemirovsky, Phys. Rev. D 28, 181 (1983).

[14] M. Tissier and N. Wschebor, Phys. Rev. D 82, 101701 (2010).

[15] M. Peláez, U. Reinosa, J. Serreau, M. Tissier, and N. Wschebor, Phys. Rev. D 96, 114011 (2017).

[16] H. J. Munczek and D. W. McKay, Phys. Rev. D 39, 888 (1989); 46, 5209(E) (1992).

[17] J. Praschifka, R. T. Cahill, and C. D. Roberts, Int. J. Mod. Phys. A 04, 4929 (1989).

[18] A. G. Williams, G. Krein, and C. D. Roberts, Ann. Phys. (N.Y.) 210, 464 (1991).

[19] L. von Smekal, P. A. Amundsen, and R. Alkofer, Nucl. Phys. A529, 633 (1991).

[20] P. Jain and H. J. Munczek, Phys. Rev. D 48, 5403 (1993).

[21] M. R. Frank and C. D. Roberts, Phys. Rev. C 53, 390 (1996).

[22] P. Maris and C. D. Roberts, Phys. Rev. C 56, 3369 (1997).

[23] P. Maris, C. D. Roberts, and P. C. Tandy, Phys. Lett. B 420, 267 (1998).

[24] P. Maris and P. C. Tandy, Phys. Rev. C 60, 055214 (1999).

[25] R. Alkofer, P. Watson, and H. Weigel, Phys. Rev. D 65, 094026 (2002).

[26] S. x. Qin, L. Chang, Y. x. Liu, C. D. Roberts, and D. J. Wilson, Phys. Rev. C 84, 042202 (2011).

[27] L. Chang and C. D. Roberts, Phys. Rev. C 85, 052201 (2012).

[28] L. Chang, I. C. Cloët, J. J. Cobos-Martínez, C. D. Roberts, S. M. Schmidt, and P. C. Tandy, Phys. Rev. Lett. 110, 132001 (2013).

[29] L. Chang, I. C. Cloët, C. D. Roberts, S. M. Schmidt, and P. C. Tandy, Phys. Rev. Lett. 111, 141802 (2013).

[30] E. Rojas, B. El-Bennich, and J. P. B. C. de Melo, Phys. Rev. D 90, 074025 (2014).

[31] K. Raya, L. Chang, A. Bashir, J. J. Cobos-Martinez, L. X. Gutiérrez-Guerrero, C. D. Roberts, and P. C. Tandy, Phys. Rev. D 93, 074017 (2016).

[32] B. El-Bennich, G. Krein, E. Rojas, and F. E. Serna, FewBody Syst. 57, 955 (2016).

[33] B. El-Bennich, M. A. Paracha, C. D. Roberts, and E. Rojas, Phys. Rev. D 95, 034037 (2017).

[34] F. F. Mojica, C. E. Vera, E. Rojas, and B. El-Bennich, Phys. Rev. D 96, 014012 (2017).

[35] C. Shi and I. C. Cloët, Phys. Rev. Lett. 122, 082301 (2019).

[36] F. E. Serna, R. Correa da Silveira, J. J. Cobos-Martínez, B. El-Bennich, and E. Rojas, Eur. Phys. J. C 80, 955 (2020).

[37] S. x. Qin and C. D. Roberts, arXiv:2009.13637.

[38] I. C. Cloët, G. Eichmann, B. El-Bennich, T. Klahn, and C. D. Roberts, Few-Body Syst. 46, 1 (2009).

[39] G. Eichmann, R. Alkofer, A. Krassnigg, and D. Nicmorus, Phys. Rev. Lett. 104, 201601 (2010).
[40] I. G. Aznauryan, A. Bashir, V. Braun, S. J. Brodsky, V. D. Burkert, L. Chang, C. Chen, B. El-Bennich, I. C. Cloët, and P. L. Cole et al., Int. J. Mod. Phys. E 22, 1330015 (2013).

[41] J. Segovia, B. El-Bennich, E. Rojas, I. C. Cloët, C. D. Roberts, S. S. Xu, and H. S. Zong, Phys. Rev. Lett. 115, 171801 (2015).

[42] G. Eichmann, C. S. Fischer, and H. Sanchis-Alepuz, Phys. Rev. D 94, 094033 (2016).

[43] G. Eichmann, H. Sanchis-Alepuz, R. Williams, R. Alkofer, and C. S. Fischer, Prog. Part. Nucl. Phys. 91, 1 (2016).

[44] H. Sanchis-Alepuz and R. Williams, Comput. Phys. Commun. 232, 1 (2018).

[45] C. Chen, B. El-Bennich, C. D. Roberts, S. M. Schmidt, J. Segovia, and S. Wan, Phys. Rev. D 97, 034016 (2018).

[46] C. Chen, Y. Lu, D. Binosi, C. D. Roberts, J. RodríguezQuintero, and J. Segovia, Phys. Rev. D 99, 034013 (2019).

[47] K. D. Bednar, I. C. Cloët, and P. C. Tandy, Phys. Lett. B 782, 675 (2018).

[48] S. x. Qin, C. D. Roberts, and S. M. Schmidt, Few-Body Syst. 60, 26 (2019).

[49] C. Chen, G. I. Krein, C. D. Roberts, S. M. Schmidt, and J. Segovia, Phys. Rev. D 100, 054009 (2019).

[50] B. Podolsky, Phys. Rev. 62, 68 (1942).

[51] B. Podolsky and C. Kikuchi, Phys. Rev. 65, 228 (1944).

[52] R. Bufalo, B. M. Pimentel, and G. E. R. Zambrano, Phys. Rev. D 86, 125023 (2012).

[53] C. A. P. Galvão and B. M. Pimentel Escobar, Can. J. Phys. 66, 460 (1988).

[54] R. R. Cuzinatto, C. A. M. de Melo, and P. J. Pompeia, Ann. Phys. (Amsterdam) 322, 1211 (2007).

[55] R. Bufalo, B. M. Pimentel, and G. E. R. Zambrano, Phys. Rev. D 83, 045007 (2011).

[56] C. R. Ji, A. T. Suzuki, J. H. O. Sales, and R. Thibes, Eur. Phys. J. C 79, 871 (2019).

[57] C. S. Fischer, A. Maas, and J. M. Pawlowski, Ann. Phys. (Amsterdam) 324, 2408 (2009).

[58] R. Alkofer, M. Q. Huber, and K. Schwenzer, Phys. Rev. D 81, 105010 (2010).

[59] D. Dudal, J. A. Gracey, S. P. Sorella, N. Vandersickel, and H. Verschelde, Phys. Rev. D 78, 065047 (2008).

[60] A. C. Aguilar and A. A. Natale, J. High Energy Phys. 08 (2004) 057.

[61] A. C. Aguilar, D. Binosi, and J. Papavassiliou, Phys. Rev. D 78, 025010 (2008).

[62] A. C. Aguilar, D. Binosi, and J. Papavassiliou, Phys. Rev. D 86, 014032 (2012).

[63] A. Cucchieri and T. Mendes, Proc. Sci. LATTICE2007 (2007) 297 [arXiv:0710.0412].

[64] A. Cucchieri and T. Mendes, Phys. Rev. Lett. 100, 241601 (2008).

[65] O. Oliveira and P. J. Silva, Phys. Rev. D 79, 031501 (2009).

[66] M. R. Pennington and D. J. Wilson, Phys. Rev. D 84, 119901 (2011).

[67] O. Oliveira and P. J. Silva, Phys. Rev. D 86, 114513 (2012).

[68] I. L. Bogolubsky, E. M. Ilgenfritz, M. Müller-Preussker, and A. Sternbeck, Phys. Lett. B 676, 69 (2009). 
[69] A. Ayala, A. Bashir, D. Binosi, M. Cristoforetti, and J. Rodríguez-Quintero, Phys. Rev. D 86, 074512 (2012).

[70] S. Strauss, C. S. Fischer, and C. Kellermann, Phys. Rev. Lett. 109, 252001 (2012).

[71] M. Q. Huber, Phys. Rev. D 91, 085018 (2015).

[72] A. K. Cyrol, L. Fister, M. Mitter, J. M. Pawlowski, and N. Strodthoff, Phys. Rev. D 94, 054005 (2016).

[73] P. Boucaud, F. De Soto, K. Raya, J. Rodríguez-Quintero, and S. Zafeiropoulos, Phys. Rev. D 98, 114515 (2018).

[74] B. W. Mintz, L. F. Palhares, G. Peruzzo, and S. P. Sorella, Phys. Rev. D 99, 034002 (2019).

[75] D. Dudal, O. Oliveira, and P. J. Silva, Ann. Phys. (Amsterdam) 397, 351 (2018).

[76] A. C. Aguilar, F. De Soto, M. N. Ferreira, J. Papavassiliou, J. Rodríguez-Quintero, and S. Zafeiropoulos, Eur. Phys. J. C 80, 154 (2020).

[77] P. J. Gunkel, C. S. Fischer, and P. Isserstedt, Eur. Phys. J. A 55, 169 (2019).

[78] P. J. Gunkel and C. S. Fischer, arXiv:2012.01957.

[79] M. Q. Huber, Phys. Rev. D 101, 114009 (2020).

[80] C. S. Fischer, P. Watson, and W. Cassing, Phys. Rev. D 72 , 094025 (2005).

[81] A. Krassnigg, Proc. Sci. CONFINEMENT8 (2008) 075 [arXiv:0812.3073].

[82] A.C. Aguilar, D. Binosi, J. Papavassiliou, and J. Rodríguez-Quintero, Phys. Rev. D 80, 085018 (2009).

[83] G. Curci and R. Ferrari, Nuovo Cimento A 32, 151 (1976).

[84] R. Alkofer, C. S. Fischer, F. J. Llanes-Estrada, and K. Schwenzer, Ann. Phys. (Amsterdam) 324, 106 (2009).

[85] A. Kizilersu and M. R. Pennington, Phys. Rev. D 79, 125020 (2009).

[86] A. Bashir, R. Bermúdez, L. Chang, and C. D. Roberts, Phys. Rev. C 85, 045205 (2012).

[87] E. Rojas, J. P. B. C. de Melo, B. El-Bennich, O. Oliveira, and T. Frederico, J. High Energy Phys. 10 (2013) 193.

[88] E. Rojas, B. El-Bennich, J. P. B. C. De Melo, and M. A. Paracha, Few-Body Syst. 56, 639 (2015).
[89] A. C. Aguilar, D. Binosi, D. Ibañez, and J. Papavassiliou, Phys. Rev. D 90, 065027 (2014).

[90] R. Williams, Eur. Phys. J. A 51, 57 (2015).

[91] M. Peláez, M. Tissier, and N. Wschebor, Phys. Rev. D 92 , 045012 (2015).

[92] S. Jia and M. R. Pennington, Phys. Rev. D 94, 116004 (2016).

[93] R. Williams, C. S. Fischer, and W. Heupel, Phys. Rev. D 93, 034026 (2016).

[94] A. Sternbeck, P. H. Balduf, A. Kızılersu, O. Oliveira, P. J. Silva, J. I. Skullerud, and A. G. Williams, Proc. Sci., LATTICE2016 (2017) 349 [arXiv:1702.00612].

[95] A. C. Aguilar, J.C. Cardona, M. N. Ferreira, and J. Papavassiliou, Phys. Rev. D 98, 014002 (2018).

[96] F. E. Serna, C. Chen, and B. El-Bennich, Phys. Rev. D 99, 094027 (2019).

[97] L. Albino, A. Bashir, L. X. G. Guerrero, B. E. Bennich, and E. Rojas, Phys. Rev. D 100, 054028 (2019).

[98] O. Oliveira, W. de Paula, T. Frederico, and J. P. B. C. de Melo, Eur. Phys. J. C 79, 116 (2019).

[99] O. Oliveira, T. Frederico, and W. de Paula, Eur. Phys. J. C 80, 484 (2020).

[100] F. Gao and J. M. Pawlowski, Phys. Rev. D 102, 034027 (2020).

[101] N. Nakanishi, Phys. Rev. 138, B1182 (1965).

[102] C. S. Fischer and R. Williams, Phys. Rev. Lett. 103, 122001 (2009).

[103] M. Tanabashi et al. (Particle Data Group), Phys. Rev. D 98, 030001 (2018).

[104] C. T. H. Davies, C. McNeile, E. Follana, G. P. Lepage, H. Na, and J. Shigemitsu, Phys. Rev. D 82, 114504 (2010).

[105] S. Aoki et al. (Flavour Lattice Averaging Group), Eur. Phys. J. C 80, 113 (2020).

[106] J. P. B. C. de Melo, K. Tsushima, B. El-Bennich, E. Rojas, and T. Frederico, Phys. Rev. C 90, 035201 (2014). 